\title{
Populismo punitivo y extrema derecha en el espacio ibérico
}

\section{Punitive populism and far right in the Iberian space}

\author{
Alfonso A. López-Rodríguez \\ Universidad de Santiago de Compostela, España \\ alfonsoantonio.lopez@usc.es \\ https://orcid.org/0000-0003-3012-5667 \\ Álvaro González-Gómez \\ Universidad de Santiago de Compostela, España \\ alvaro.gonzalez@rai.usc.es \\ https://orcid.org/0000-0002-6177-4662 \\ Serafín González-Quinzán \\ Universidad de Santiago de Compostela, España \\ serafin.gonzalez.quinzan@usc.es \\ https://orcid.org/0000-0002-7746-5944
}

Recibido: 30/03/2021 Revisado: 23/04/2021 Aceptado: 01/06/2021 Publicado: 01/09/2021

\section{Resumen}

La entrada de formaciones de extrema derecha populista en las instituciones de España y Portugal supone un cambio cualitativo de sus sistemas políticos. Una cuestión relevante es el uso electoralista de la estrategia del populismo punitivo, una herramienta ya utilizada de manera extensiva por otras formaciones más veteranas dentro de esa familia ideológica. La cuestión que aborda este texto es en qué grado han utilizado esta estrategia y si han realizado alguna modulación particular de la misma dentro de sus manifiestos electorales vigentes durante los comicios de entrada en las cámaras parlamentarias de cada uno de los países ibéricos.

\section{Palabras clave}

Extrema derecha, populismo punitivo, Europa, Península Ibérica.

Forma sugerida de citar: López-Rodríguez, A.A., González-Gómez, A., \& González-Quinzán (2021). Populismo punitivo y extrema derecha en el espacio ibérico. UniversitasXXI, 35, pp. 103-126. https://doi.org/10.17163/uni.n35.2021.05 


\begin{abstract}
The entry of extreme right-wing populist formations into the institutions of Spain and Portugal represents a qualitative change in their political systems. One relevant issue is the electoral use of the strategy of punitive populism, a tool already used extensively by other more veteran formations within this ideological family in Europe. The question addressed in this text is to what extent they have used this strategy, and whether they have made any particular modulation of it in their electoral manifestos in force during the elections for entry into the parliamentary chambers of each of the Iberian countries.
\end{abstract}

Keywords

Far right, punitive populism, Europe, Iberian Peninsula.

\title{
Introducción y estado de la cuestión
}

El 6 de octubre de 2019 se consuma el fin del excepcionalísimo ibérico (Simón, 2005). Esto es, la ausencia de partidos de extrema derecha dentro de las cámaras legislativas de Portugal y España, en contraste con lo que viene a ser habitual en los países europeos, desde finales de la década de 1990. Chega! obtiene representación parlamentaria en Portugal. En abril de ese mismo año, Vox, en España, ya había entrado en la Cámara Baja. Además, en noviembre obtendría un incremento de apoyo electoral, permitiendo su entrada en el Senado al tiempo que aumenta su presencia en el Congreso de los Diputados. En ese año 2019, sendas formaciones de extrema derecha populista (en adelante EDP), experimentan un salto cualitativo, apoyado en formas y estrategias nuevas, tanto de comunicación y discurso, como de proyecto. Dentro de esta estrategia, resulta un elemento sobresaliente la presencia del populismo punitivo, por la saliencia dentro de los programas y manifestaciones públicas de las citadas formaciones.

El objetivo del presente texto es ver hasta qué punto estas formaciones de EDP han utilizado el discurso del populismo punitivo dentro de sus campañas y en qué coordenadas lo han expresado. Esto es relevante dado que este tipo de práctica se ha hecho usual dentro de las formaciones de esta na- 
turaleza (Hough, 2008; Stojarová, 2018). Asimismo, resulta pertinente en tanto que las citadas formaciones gozan de representación institucional y de una visibilidad pública que, hasta cierto punto, les permite introducir temas en la agenda política.

Como elemento necesario, para alcanzar el objetivo propuesto, resulta adecuado presentar a cada una de las formaciones y el contexto donde despliegan su actividad. De este modo, posteriormente, desarrollar el concepto del populismo punitivo y sus elementos constituyentes fundamentales. Con esta finalidad se describen - brevemente - las formaciones de EDP ibéricas.

Comenzando con el partido español, Vox, nace en 2013 como una escisión a la derecha del partido gobernante en aquellos momentos, el conservador Partido Popular (en adelante PP). Si bien en los primeros comicios a los que se presentó obtuvo unos resultados testimoniales, varios factores a lo largo de 2019 ayudaron a que realizase un salto cualitativo. Por un lado, los impactos de la Gran Recesión (Nikolaus, 2010) en España y las medidas de la Troika ${ }^{1}$ causaron un malestar en amplios sectores de la población a lo largo de todo el espectro ideológico, si bien por el lado derecho tardaron más en manifestarse política y electoralmente. Por otro, el partido en el gobierno se enfrentó a un triple desgaste: el propio de desempeñar las tareas de gobierno en un escenario socioeconómico adverso, la crisis de la declaración de independencia de Cataluña y los casos de corrupción que asedian a la formación. Este desgaste partidista, paralelo al del líder de la formación y jefe de gobierno, Mariano Rajoy, permitió a Vox el acceso al caladero de votantes anteriormente monopolizado por el PP.

Por su parte, el caso de Chega! presenta similitudes, en tanto que nace también como escisión del partido hegemónico de la derecha portuguesa el PSD. ${ }^{2}$ El caso luso es relevante porque la formación se constituye en abril de 2019 y en octubre de ese mismo año ya obtiene representación en la cámara legislativa nacional. La aparición de la formación de EDP portuguesa tiene razones parecidas a la española. Portugal ha sido un país también castigado por la Gran Recesión de 2008, teniendo que recibir un rescate explícito

1 Con este nombre se conoce a los tres órganos: Comisión Europea, Banco Central Europeo y Fondo Monetario internacional. Estos, en conjunto, funcionan como grupo de decisión para las políticas de rescate de los países miembros de la Unión Europea, en especial tras las crisis de deuda soberana generadas por el impacto de la Gran Recesión en la región.

2 Siglas del Partido Social Demócrata. Cabe señalar que el partido se ubica, a pesar de lo que indica el nombre, en el espectro ideológico del centro derecha. 
en 2011, durante el gobierno del PSD. Eso provoca un alto desgaste para el Ejecutivo, a lo largo de los siguientes años, por causa de los elevados costes sociales de las medidas impuestas para el acceso al rescate financiero.

De este modo, en 2015, el candidato del PSD y jefe de gobierno, Pedro Passos Coelho, lidera la lista más votada, pero no es capaz de renovar como Primer Ministro, accediendo al gobierno Antonio Costa del Partido Socialista. Este hecho provoca una crisis de liderazgo dentro del PSD, algo acusa mucho esta formación, dado el peso del líder y el carácter carismático que suelen tener (Magone, 1998). En 2017 André Ventura, en aquel momento candidato del PSD en las elecciones locales, realiza unas declaraciones polémicas en torno a la población de etnia gitana (Martíns Carvalho, 2017). Las alusiones a las minorías y el endurecimiento del código penal serán constantes una vez que sale del PSD y funda Chega!

Cabe señalar que esta formación de EDP hereda el modelo originario de su formación matriz, en tanto que el peso del líder resulta crucial. En parte porque es el único diputado representado, por el momento, en la Asamblea de la República.

Los discursos centrados en el populismo punitivo enraízan el contexto de malestar social (Berman, 2019), en el caso de los países ibéricos estos han sufrido un fuerte impacto en sus tejidos social y económico con la Gran Recesión, seguida de la crisis del euro, y aún sufren de sus secuelas. La deconstrucción de los estados sociales europeos dentro del modelo de integración económica hiperglobalizado (Rodrik, 2012) provocó la expansión de las desigualdades sociales (Piketty, 2015). Este proceso, tanto a nivel local como global ha desembocado en un manifiesto malestar con la globalización (Stiglitz, 2003).

La Gran Recesión de 2008 implica para los países de la Unión Europea el descubrimiento de las limitaciones del modelo de manera única (Stiglitz, 2016; Chari et al., 2020). Las deficiencias del modelo presionaron a las economías periféricas de la zona (como el caso de las naciones ibéricas) a realizar un ajuste por medio de la devaluación interna (Rísquez, 2016). Esto es, al no poder aplicar una devaluación monetaria, se devalúan a las personas, más concretamente las rentas salariales como mecanismo para ganar competitividad en los mercados nacionales impulsando las exportaciones. Cabe destacar, que tanto España como Portugal ya tenía unos niveles salariales bajos en comparación con la media europea. Esto, a su vez, motivó movilizaciones sociales de protesta, junto con un empobrecimiento y precarización 
de las condiciones de vida, con el incremento de situación de riesgo de pobreza y exclusión social (Tejero-Pérez, 2017).

Asimismo, este contexto va a verse severamente agravado por la emergencia sanitaria de la COVID-19, los países ibéricos son particularmente frágiles dada su exposición a la coyuntura global (que afecta al flujo de turistas) y a la dependencia de los mercados europeos en el ámbito comercial. En el caso de España, una parte sustancial de su economía depende de la actividad turística, prácticamente inexistente desde el comienzo de la pandemia (Picazo \& Gil-Pareja, 2020). Esta situación se ha concretado en una creciente inseguridad laboral y económica provocando la expansión de grupos sociales dentro de la dinámica de la modernidad excluyente. Tanto en el contexto global, como en el Viejo Continente, con la expansión de la categoría de los trabajadores pobres (Marx, 2020). Además, se constata que los incrementos en las desigualdades socioeconómicas, la deficiente red del estado de bienestar y la falta de oportunidades laborales ha provocado un repunte de ciertos delitos relativos a la propiedad, especialmente robos y hurtos.

Este tipo de hechos, magnificados por los medios de comunicación, conforman corrientes de opinión en torno a la inseguridad y el temor a la victimización. Esto constituye un contexto donde, si bien dentro de ratios de criminalidad bajas, se genera un clima de inseguridad para los llamados insiders. ${ }^{3}$ En ambos países se ha ido aquilatando un marco donde una creciente población se ve más y más excluida y con escasas oportunidades de mejora, frente a ellos, estratos de población incluida, o integrada, temen ser victimizados, es decir, el temor a ser víctimas de algún tipo de delito.

Es en un contexto de este tipo donde los discursos como el populismo punitivo funcionan con mayor eficacia, como promesa o bálsamo de recupe-

3 La dinámica insider/outsider (Carrabine et al., 2014, p. 346) es utilizada en criminología como una línea de distinción del tipo "nosotros/ellos". En el contexto del presente texto se adopta la nomenclatura inglesa y la misma distinción. Al distinguir entre ciudadanos insiders y outsiders se alude al criterio de una socialización normalizada y a un respeto de las normas tanto sociales como jurídicas. De este modo, se entiende que el ciudadano insider está inserto en la sociedad, integrado en ella, y realiza una actividad dentro de los canales considerados normales, con respeto a las normas tanto formales como informales. Además, mantiene comportamiento considerador normal dentro del colectivo social de pertenencia. En cambio, el outsider sería la persona que, voluntariamente o no, se encuentra en los márgenes de la sociedad, no respetando completamente el conjunto de normas sociales y formales. Sea por desconocimiento, incapacidad o por propia voluntad. Cabe distinguir, de este modo, aquellas personas que están en una situación de exclusión social por casusas socioeconómicas (como por ejemplo la falta de oportunidades) y desean normalizarse/integrarse. Por otro lado, están los que voluntariamente se autoexcluyen pudiendo en casos, formar parte de subculturas violentas o delictivas. 
ración de la ley y el orden, mediante un código penal endurecido y expandido, mediante el efecto tipificación de nuevos delitos, o el agravamiento de los ya existentes.

\section{Hacia una caracterización del populismo punitivo}

El nacimiento del populismo punitivo aparece en la década de 1980, dentro del ámbito anglosajón, vinculado a las políticas de seguridad y orden público. Entronca con un enfoque neoconservador o "actuarial" (McLaughlin \& Muncie, 2011) de las políticas de seguridad que se asientan en unos pilares fundamentales. Por un lado, la hipótesis que el incremento en las penas genera un efecto de desistimiento en los potenciales delincuentes. Por otro, la idea que las penas reforzadas fortalecen a su vez el consenso moral mayoritario. Además, la aplicación de este tipo de medidas redunda en incremento de apoyo electoral. En todo este razonamiento está implícito la dinámica entre los insiders frente a los outsiders (Carrabine, et al., 2014). Este modelo de políticas busca, en último término, el cohesionar a la comunidad de personas plenamente integradas frente a los colectivos que se consideran en los márgenes de la sociedad, en situaciones de exclusión o autoexclusión. Como indica David Garland (2005, p. 300) se trata de construir la imagen de un "otro peligroso" y señalarlo como culpable o potencialmente culpable de los delitos cometidos, facilitando el consenso para el incremento de las penas. Consecuentemente, se incrementa la sensación de seguridad de los insiders. Es, por tanto, un modelo de políticas vinculado a la "Revolución Conservadora" (Herrendorf, 1988; Huguet, 2005) y a los partidos tradicionales de ese ámbito del espectro ideológico.

Así, desde la década de 1980 en adelante, se pasa del modelo de gestión de la delincuencia fundado en el welfare para adentrarse en el paradigma del warfare (Larrauri, 2018, p. 213), se sustituye el objetivo de la reinserción, por el de la punición e inocuización del delincuente. De manera que se abandonan, en el ámbito de las políticas criminales los objetivos de reinserción de los delincuentes como la norma dentro de este tipo de políticas públicas, pasando a ser la excepción. Además, se posterga la búsqueda de las causas estructurales de las actividades delictivas; sean estas la marginación, la exclusión social, una socialización disfuncional, etc. Se parte de entender que el individuo es un agente racional puro y que todos somos delincuentes, si 
tenemos la oportunidad, de acuerdo con el paradigma de las "ventanas rotas" (McLaughlin \& Muncie, 2011, p. 542). Por lo tanto, la delincuencia emerge por la insuficiente vigilancia y control social, por ello se deben intensificar las acciones policiales y endurecer los códigos penales. El planteamiento parte de entender que existe un déficit de seguridad (en términos de orden público) y ha de resolverse de manera contundente.

De este modo, las propuestas conservadoras en materia de seguridad se inhiben en la búsqueda de causas profundas de la delincuencia, adoptando un enfoque preferentemente curativo y postergando las medidas preventivas. El delincuente es visto como un actor racional que se limita a aprovechar las oportunidades, por lo que las soluciones no están en los orígenes y disfunciones de la socialización, la exclusión social o la falta de oportunidades, si no en medidas concretas que favorezcan el desistimiento del curso de acción delictivo. Por un lado, reforzar las normas, en concreto con penas intimidatorias en relación con la gravedad del delito cometido, de manera que los beneficios de la comisión de un acto delictivo no sean directos, evidentes y ventajosos. Además de fomentar las medidas que dificultan la comisión, de modo que perpetrar el delito sea profundamente antieconómico dentro de un balance coste-beneficio.

Como resultado de este cambio en las políticas de seguridad, el código penal se ha convertido en un arma más de las luchas en la arena política. Se contempla una instrumentalización creciente del código penal y las políticas securitarias como una baza para la maximización del voto en sociedades donde se pierde la cohesión social y crece el miedo a la victimización. De esta manera, termina por construirse un contexto donde casi todas las formaciones políticas presentan su proyecto de política penal con tipificaciones diferentes para los delitos que consideran más relevantes, o que generan el mayor "pánico moral" (Cohen, 2002) dentro de sus potenciales votantes. De modo que termina por asentarse un populismo punitivo por el lado de la oferta, que trata de captar a los votantes que demandan mayor seguridad.

Esta corriente, en principio vinculada a opciones políticas conservadoras, se ha ido extendiendo por todo el espectro de los sistemas de partidos, prendiendo de manera particularmente fuerte entre los del extremo derecho (Liang, 2007). Además, cabe señalar (Mudde, 2019) que la ideología de la ley y el orden se enmarca dentro de un contexto fuertemente autoritario dentro de los movimientos de extrema derecha. Más concretamente, desde comienzos del siglo XXI, pero especialmente tras el comienzo de la crisis eco- 
nómica de 2008, se ha establecido en el Viejo Continente una "ideología de la ley y el orden" adaptada, en un primer momento, por los partidos tradicionales de derecha e izquierda (Díez-Ripollés, 2004). Se adopta, por parte de los citados partidos, una retórica alarmista y de matriz populista.

En este contexto, la seguridad ciudadana se convierte, en España y Portugal, en un interés electoralista, teniendo en principio una importancia marginal, que se incrementa con el tiempo. Entre las principales causas que señalaban los partidos españoles a principios del siglo: la reducción de recursos policiales y la falta de inversiones en el ámbito securitario. Dentro de esta coyuntura se desarrollan algunos de los elementos inherentes al populismo punitivo, como es la penalidad expresiva y una legislación penal simbólica (Arrieta-Ruiz, 2018). Parte de la tendencia a generar un modelo penal emocional, a menudo vinculado con una modificación de los tipos penales desordenada y precipitada. Esto está motivado por la presión de la opinión pública del momento ante algún caso de actualidad, particularmente sensible, implicando un fuerte componente emocional (García-Pablos, 2014). Estas corrientes de opinión presionan hacia un cambio legislativo represivo como mecanismo de atracción electoral. De este modo, aparece otro elemento fundacional del populismo punitivo: el retorno de la víctima (Larrauri, 2018). Frente a las propuestas criminológicas que parten de la Ilustración, el modelo populista recupera el protagonismo de la víctima a la que ha de resarcirse. Se entiende que la víctima ha de restaurarse, sentirse desagraviada del delito sufrido, por medio de una pena ejemplarizante al delincuente. Esta pena ha de ser no solo dura, si no estigmatizante, dado que ha de representar también un desagravio para la comunidad en la que ha tenido lugar y funcionar como un elemento de cohesión colectiva.

Se consuma, de esta manera, una huida del derecho penal garantista hacia una escalada punitiva dentro de una retórica populista como estrategia electoral productiva. En opinión de Garland (2005) este tipo de aproximaciones suponen abordar el fenómeno del delito únicamente desde los síntomas, pero desechando desentrañar las causas. Construyéndose así un modelo de contención cuantitativa, pero no de resolución cualitativa. Esto es, una aproximación preventiva que opere sobre las causas. El mecanismo para afrontar el delito es el aumento de la actividad policial y el incremento de la regresividad penal, con los efectos de tipificación y de cumplimiento íntegro a la cabeza. El objetivo: crear un fuerte efecto disuasor del delito entre los outsiders (los otros peligrosos) y con la aquiescencia acrítica del insider. 
De este modo, se concreta en Europa un ciclo autoritario sincrónico al desmantelamiento del Estado de Bienestar, culminando el reemplazo del Estado asistencial-preventivo por un Estado residual-punitivo. Este modelo pretende acabar con la criminalidad en sus expresiones más comunes y molestas mediante estrategias de represión policial y penal. Sin embargo, obvia otros tipos, más lesivos socialmente en el largo plazo, con un mayor daño social y un coste elevado para la legitimidad del sistema político. Son los tipos penales vinculados a la corrupción política y corporativa, los delitos fiscales o de tráfico de influencias, en resumen, aquellos conocidos como de guante blanco (Cuervo- García, 2019) usualmente perpetrados por élites sociales.

Tanto en los programas, como en la práctica gubernativa, este populismo punitivo tiende al abuso de las penas de prisión, cada vez más largas, como elemento neutralizador del delito. Este mecanismo, junto con la presencia policial, busca generar un sentimiento de protección y seguridad. Todo ello dentro de un escenario de solidaridad con las víctimas, protagonistas emocionales del hecho delictivo. Esto supone un cambio en la misma concepción de Estado, para el ciudadano insider no es un ente que amenaza la libertad individual, sino un aliado que lo defiende de los riesgos de una sociedad desestructurada. El Estado termina por convertirse en el compañero y protector que evita la victimización de los ciudadanos integrados, de aquellos que ocupan el lugar correcto de la sociedad.

De este modo, el populismo punitivo termina cristalizando, no solo en un discurso y unas políticas a él vinculadas, sino también en una ideología de la seguridad ciudadana. Es, en suma, una estrategia integral que aglutina el discurso político, las políticas públicas y una manera de entender los fenómenos sociales relacionados con la seguridad y la delincuencia. Este paradigma implica, de un modo necesario, la instrumentalización del código penal como herramienta que facilita la obtención de ventajas políticas y electorales. Se consuma así el abandono, de dicho código, como mecanismo para la resolución de conflictos sociales concernientes a la seguridad del colectivo ciudadano. Este es un factor que implica el riesgo de erosión de principios liberales propios de los sistemas liberal representativos o poliarquías (Mounk, 2019), o, al menos, su vaciado de su contenido originario.

El Derecho Penal pasa a ser un elemento subalterno de las presiones políticas, pasando a ser mero instrumento técnico, con bajos costes, que permite mostrar una apariencia de eficacia estatal. Permite manufacturar una tranquilidad y calmar las expectativas de la población en relación con deter- 
minados delitos, muy vinculados con la criminalidad común. De modo que el populismo punitivo termina por constituir, en el ámbito de los hechos, no un modelo que facilite una seguridad objetiva, si no que busca crear una sensación subjetiva de seguridad dentro de una parte sustancial del electorado.

Puede concluirse que durante el siglo XXI los issues relativos a la seguridad ciudadana y la delincuencia han sido apropiados por los partidos de EDP en Europa. Esto se ha producido de tal modo que ha terminado por ser uno de los temas preferentes o "cliché" (Davies \& Jackson, 2008, p. 95) de este tipo de formaciones, junto a los más clásicos como la inmigración, la identidad o la soberanía. Especialmente relacionando la inmigración con temas relativos a la "ley y el orden". Todo lo anterior motiva que su adopción y adaptación del populismo punitivo se materializa en un discurso centrado en la creación de sistemas penales represivos y particularmente gravosos para los no nacionales.

Por último, se puede realizar un sumario de los elementos fundamentales del populismo punitivo en una tabla como la siguiente. En ella se explicitan los ítems clave de este tipo de estrategia política.

Tabla 1

\section{Elementos constitutivos del populismo punitivo}

\begin{tabular}{|l|}
\hline Penalidad expresiva \\
\hline Protagonismo de la víctima \\
\hline Incapacitación-inocuización del delincuente \\
\hline Penas agravadas y estigmatizantes \\
\hline Electoralismo \\
\hline Temor a la victimización \\
\hline Criminalización del "otro peligroso" \\
\hline Prevención situacional (énfasis en la acción policial y los obstáculos al delito) \\
\hline Instrumentalización del Código Penal (efecto tipificación) \\
\hline
\end{tabular}

Fuente: Elaboración propia a partir de Garland (2005), Carrabine et al. (2014) y Larrauri (2018).

La tabla anterior, además de un sumario de los principales elementos que constituyen el modelo de populismo punitivo, servirá de parámetro de contraste con las medidas que contemplan los manifiestos electorales de los 
dos partidos de EDP ibéricos. Una vez realizada la presentación del tema, justificando su pertinencia y relevancia, y tras haber descrito la situación donde se inscribe el objeto de estudio y haber definido el marco teórico general, se presenta el marco metodológico propio de la investigación.

\section{Material y método}

Como material fundamental, esto es, como objeto de estudio, se ha optado por el análisis de los manifiestos electorales de las formaciones estudiadas, asumiendo del paradigma de análisis documental (Valles, 1999). Más en concreto los que estaban vigentes en los comicios de entrada en las cámaras legislativas de sus respectivos países. Así se podrá comparar las similitudes y divergencias que, respecto a la adopción del discurso del populismo punitivo, presentan ambos casos en el momento de realizar el salto cualitativo hacia la representación institucional.

En relación con el método concreto, dado el carácter del objeto, se ha optado por un análisis del contenido que abarque una cuantificación de los ítems de ambos manifiestos como una evaluación de su contenido (Díaz-Herrera, 2018). La lógica se encuadra dentro de una secuencia de tres estadios. En primer lugar, una cuantificación de los bloques temáticos independientes, seguidamente una reorganización de los mismos en función al criterio de similitud. De ese primer estadio pueden inferirse algunas similitudes y diferencias generales. Este momento tiene la funcionalidad de describir el contexto general donde se incardinan dichos manifiestos.

El segundo paso es la reordenación en un bloque temático homogéneo de las propuestas de cada uno de los manifiestos, relativas al populismo punitivo. Este paso implica poder discriminar aquellas propuestas que pertenecen inequívocamente al ámbito relevante de análisis, facilitando su comparación y peso relativo.

De este modo, en el tercer estadio, puede realizarse una comparación estandarizada en términos cuantitativos, pudiendo establecer el peso, en relación con el resto de propuestas. Además, se facilita la comparación en términos cualitativos aludiendo a sus enunciados y contenidos.

El objetivo final es responder a la cuestión principal: han utilizado, o no, las formaciones de EDP ibéricas un formato de propuestas propio del populismo punitivo. En caso afirmativo, resta la cuestión de si han realizado una adaptación propia a su entorno o se han mantenido en manifestaciones y propuestas meramente genéricas. 


\section{Análisis y resultados}

Como se indicó en la sección anterior, se procede a organizar de una manera descriptiva los resultados de aglutinar los grandes ejes temáticos para los manifiestos de cada una de las fuerzas políticas. Para el caso de la formación española se obtiene una tabla como la siguiente. En ella el peso indica el porcentaje de propuestas respecto al total de las contenidas dentro del manifiesto.

\section{Tabla 2}

\section{Manifiesto electoral Vox}

\begin{tabular}{|l|r|l|}
\hline \multicolumn{3}{|c|}{$\mathbf{1 0 0}$ medidas para la España Viva } \\
\hline \multicolumn{1}{|c|}{ Nombre del Bloque } & \multicolumn{1}{c|}{ Peso } & \multicolumn{1}{c|}{ Contenido principal } \\
\hline España, unidad y soberanía & $10.0 \%$ & Unidad nacional y recentralización \\
\hline Ley electoral y transparencia & $3.0 \%$ & Elecciones y corrupción \\
\hline Defensa, seguridad y fronteras & $9.0 \%$ & $\begin{array}{l}\text { Limitar llegada, dificultar permanencia y facilitar } \\
\text { expulsión de inmigrantes }\end{array}$ \\
\hline Economía y recursos & $21.0 \%$ & $\begin{array}{l}\text { Incremento inversión y refuerzo de la frontera. } \\
\text { Islamismo como problema }\end{array}$ \\
\hline Salud & $5.0 \%$ & Reducción del gasto público y reforma Fiscal \\
\hline Educación y cultura & $10.0 \%$ & $\begin{array}{l}\text { Promocentralización de valores españoles. Homogeneidad de } \\
\text { programas educativos. Cheque escolar y PIN Parental }\end{array}$ \\
\hline Vida y familia & $7.0 \%$ & Pro-vida. Antifeminismo \\
\hline Libertades y Justicia & $14.0 \%$ & Antiterrorismo. Endurecimiento del CP(Código Penal). \\
\hline Europa e internacional & $5.0 \%$ & Euroescepticismo Recuperación de la soberanía nacional \\
\hline
\end{tabular}

Fuente: Elaboración propia a partir de Vox (2019).

En un marco meramente descriptivo, ya se aprecia, por un lado, la fuerte presencia de la inmigración como un tema casi transversal que es propio de la formación española. Casi la cuarta parte de las propuestas están dentro de la economía ( $21 \%$ ) lo que se explica por la situación de crisis socioeconómica que atraviesa el país desde 2008. La mayoría de las medidas se inscriben dentro de políticas de la ortodoxia neoliberal, tendente a crear un Estado de Bienestar residual (Esping-Andersen, 1996). Asimismo, hay que destacar que la presencia de medidas antiterroristas y en defensa de unidad 
nacional y la recentralización. En especial, para el tema objeto de este estudio, destacan las medidas como la 87, donde se alude explícitamente a la desaparecida banda terrorista ETA. Así como en las 84 y 85, en las que señala explícitamente a las víctimas de dicha organización violenta, al deber de ser homenajeadas, mantener su memoria y su dignidad. Esto tiene origen en la fundación del partido por personas víctimas del terrorismo de la citada organización. Además, su líder Santiago Abascal fue amenazado por la organización terrorista en el pasado. Asimismo, dicha banda tenía como fin último la independencia de los territorios vascos, lo cual es un desafío a la unidad nacional, un valor supremo para la formación de EDP. Esto explica el énfasis en el primer bloque sobre la unidad del Estado y las políticas de recentralización. Todo ello va en paralelo con una criminalización y condena rotunda de toda vía separatista, a tenor de lo manifestado en el punto 2 "Ilegalización de los partidos, asociaciones u ONG que persigan la destrucción de la unidad territorial de la Nación y su soberanía” (Vox, 2019, p. 1).

Por último, se destaca una parte final de peso escaso $(5.0 \%)$ pero donde se manifiesta un soberanismo frente las instituciones comunitarias y una vinculación con los grupos de estados más críticos con el proceso de integración.

En lo que respecta a la formación portuguesa Chega!, su manifiesto lleva el nombre de "70 medidas para levantar Portugal". 4

Tabla 3

\section{Manifiesto electoral Chega!}

\begin{tabular}{|l|c|l|}
\hline \multicolumn{1}{|c|}{ Nombre del Bloque } & Peso & \multicolumn{1}{c|}{ Contenido principal } \\
\hline $\begin{array}{l}\text { Identidad nacional y } \\
\text { familia }\end{array}$ & $14.5 \%$ & $\begin{array}{l}\text { Posición de Portugal en el mundo, familia tradicional, } \\
\text { antifeminismo }\end{array}$ \\
\hline Educación & $5.7 \%$ & $\begin{array}{l}\text { Becas escolares, movilidad de docentes, autorización } \\
\text { parental para determinados contenidos escolares }\end{array}$ \\
\hline Justicia & $17.1 \%$ & Endurecimiento del CP. Inversión en fuerzas policiales \\
\hline Seguridad & $7.1 \%$ & $\begin{array}{l}\text { Unificación cuerpos policiales, mejora de la defensa de } \\
\text { las fronteras marítimas }\end{array}$ \\
\hline Economía & $22.8 \%$ & Desregulación, privatización, reformas fiscales \\
\hline
\end{tabular}

4 Se procede a traducir del portugués (idioma original) para el resto del texto. 


\begin{tabular}{|l|l|l|}
\hline Salud & $11.5 \%$ & $\begin{array}{l}\text { No universalidad. Nuevos servicios. Residencias } \\
\text { geriátricas }\end{array}$ \\
\hline RR.II. e inmigración & $12.8 \%$ & $\begin{array}{l}\text { Rechazo pacto inmigración. Euroescepticismo. Limitar } \\
\text { llegada, dificultar permanencia, facilitar expulsión de } \\
\text { inmigrantes }\end{array}$ \\
\hline Medio Ambiente & $8.5 \%$ & Conservación forestal. Caza. Suficiencia energética \\
\hline
\end{tabular}

Fuente: Elaboración propia a partir de Chega! (2019).

Aunque con una estructura diferente, el manifiesto luso presenta unos contenidos muy similares. Cabe destacar la magnitud prácticamente similar del apartado de medidas económicas $(22.8 \%)$ que responden a un planteamiento cercano al "fundamentalismo de mercado" (Stiglitz, 2016) y que configuran un modelo de Estado de Bienestar de tipo residual como en el caso anterior. De hecho, en la propuesta lusa sí que está presente el carácter estigmatizante del recurso a la provisión estatal, como reza la propuesta 44:

En cumplimiento del principio fundamental de subsidiariedad, la entidad "Estado" serán meramente suplementarias y/o complementarias en la prestación de servicios y el suministro de productos y solo después de haber agotado todas las alternativas privadas, Se han agotado las alternativas sociales, mutualistas o cooperativas para la prestación de estos servicios. (Chega!, 2019, p. 6)

La estigmatización se vería materializada en la propuesta 46, dado que se condiciona la percepción de las prestaciones de desempleo a la realización de trabajos comunitarios, sin especificar la índole de estos. Dicha proposición contraviene la lógica prestacional de ese tipo de derechos, dentro de las políticas laborales pasivas, dado que se condiciona a realizar una contraprestación laboral.

Un elemento de comparación que trasluce similitudes es el peso de las cuestiones económicas, dado que ambos países sufrieron una fuerte crisis tras la Gran Recesión. En las diferencias, se aprecia, en el caso de España, aquellas que emergen de un elemento estructural de su sistema político. Esto es, el clivaje centro-periferia, absolutamente ausente en Portugal. La naturaleza descentralizada y multinivel de España, debida a su diversidad interna, implica que existen territorios con personalidad propia. Estos, reconocidos constitucionalmente como nacionalidades históricas, son vistos con hostili- 
dad desde una perspectiva política centralista que sostiene la formación de EDP española. Además, el conflicto centro-periferia se materializa en unos partidos que cuestionan la unidad del Estado (o, al menos la distribución del poder político en su interior) amenazando la integridad del Estado. Sin embargo, dicho clivaje, también se manifiesta en otros fenómenos como el terrorismo independentista de ETA o el proceso de independencia de Cataluña. Ante esta realidad, Vox reacciona dentro de una criminalización de este tipo de procesos, junto con una reivindicación de la unidad nacional y la recentralización estatal.

Realizada una primera comparación contextual, se aborda la estandarización de los ítems de cada manifiesto correspondiente con las dimensiones que componen el populismo punitivo. Para ello se muestra, en primer lugar, una tabla agregada, señalando su peso sobre el total y el número correspondiente a cada propuesta. Cabe señalar que en el caso español no se han integrado las propuestas relativas al terrorismo de ETA dado que es un elemento puramente endógeno del caso español, completamente ausente en el caso portugués.

Tabla 4

Bloque populismo punitivo

\begin{tabular}{|l|c|c|}
\hline Formación & $\begin{array}{c}\text { Peso } \\
\text { relativo }\end{array}$ & \multicolumn{1}{c|}{ Propuestas vinculadas } \\
\hline Vox & $19.0 \%$ & $11,15,16,17,18,19,23,24,25,26,30,31,32,86,89,90,91,92,94$ \\
\hline Chega & $18.5 \%$ & $19,20,21,22,23,24,25,30,31,57,58,59,61$ \\
\hline
\end{tabular}

Fuente: Elaboración propia.

Un primer elemento destacable resulta de la mayor dispersión de los ítems dentro del programa de Vox respecto del de Chega!. Así como representar un volumen total mayor. Adelantando lo que se verá en la siguiente tabla, una causa estriba en el mayor impacto del terrorismo en el país, tanto el de naturaleza separatista, como el vinculado al integrismo islámico. En todo caso, el peso respecto al total el manifiesto es muy similar, $19 \%$ para la formación española y un $18.5 \%$ para la lusa. La diferencia puede explicarse en algunos de los resultados que se expresarán en la tabla 5. En ella, como en la siguiente, se organizan las propuestas en relación con las dimensiones que componen el populismo punitivo. 


\section{Tabla 5 \\ Bloque populismo punitivo detallado Vox}

\begin{tabular}{|c|c|}
\hline Bloque & Propuestas vinculadas \\
\hline Penalidad expresiva & $\begin{array}{l}\text { - 89* voz de la víctima en la ejecución de la sentencia } \\
\text { - } 91 \text { soberanía judicial no amparo en organismos europeos }\end{array}$ \\
\hline Protagonismo víctima & - $89 *$ voz de la víctima, presente en todas las fases judiciales \\
\hline Inocuización & - 90 rigor de la cadena perpetua \\
\hline Penas agravadas & - 92 eliminación de los privilegios penitenciarios \\
\hline Electoralismo & - 18 persecución mafia venta ambulante \\
\hline Temor a la victimización & - 86 promulgación ley antiocupación, anti usura, legítima defensa \\
\hline Criminalización & $\begin{array}{l}\text { - } 14 \text { deportación de inmigrantes ilegales } \\
\text { - } 15 \text { deportación de inmigrantes con crímenes graves o reincidencia } \\
\text { - } 19 \text { supresión del arraigo para la regulación de inmigrantes ilegales } \\
\text { - } 23 \text { prohibición de mezquitas yihadistas } \\
\text { - } 24 \text { persecución del fundamentalismo Islámico } \\
\text { - } 25 \text { prohibición del islam en la Educación } \\
\text { - } 31 \text { Publicación de nacionalidad y origen de los delincuentes }\end{array}$ \\
\hline Prevención situacional & $\begin{array}{l}\text { - } 26 \text { refuerzo de las fronteras (Muro fronterizo, dotación a FCSE) } \\
\text { - } 33 \text { denuncia del Espacio Schengen }\end{array}$ \\
\hline Instrumentalización CP & $\begin{array}{l}\text { - } 16 \text { revisión del tipo penal contra actividad de tráfico de personas } \\
\text { - } 30 \text { introducción del atentado a la autoridad } \\
\text { - } 94 \text { tipificación como delito del despilfarro público }\end{array}$ \\
\hline
\end{tabular}

Fuente: Elaboración propia.

$\mathrm{Al}$ apreciar el contenido de la propuesta de la formación de EDP española con las nueve dimensiones del populismo punitivo, se aprecia que están presentes en su integridad. En primer lugar, cabe destacar la propuesta 89, dado que su redacción motiva su presencia en dos dimensiones de manera simultánea. Esto se justifica en que la redacción incide tanto en la presencia de la víctima como elemento protagónico en el proceso judicial como, de manera singular, en la sentencia. Esto último lo vincula profundamente a la idea de una penalidad expresiva, esencialmente en términos de resarcir y otorgar una satisfacción a la víctima. Desde el punto de vista cuantitativo, destaca el ítem de criminalización. Este implica la idea de la identificación de un "otro peligroso" como antagonista o imagen del outsider que focaliza los riesgos de victimización para los insiders. Para el caso que se analiza está completamente volcado con la figura del inmigrante, señaladamente aquel de credo 
islámico. Esto se debe tanto a la historia reciente del país, como a las presiones migratorias a las que se ha visto expuesta la frontera sur española. Lo anterior se vincula a la dimensión de la prevención situacional, que cuenta con dos propuestas que suponen el control y refuerzo de las fronteras exteriores.

En este sentido, hay que señalar que el ámbito de construcción de ese "otro peligroso" centrado en una criminalización, casi en exclusiva, del inmigrante tiene un matiz electoralista, relacionada con las crisis migratorias que ocurren en las fronteras españolas y que sirven como fuente constante para el conflicto político y el alarmismo social. En la dimensión de la penalidad expresiva se aprecia, además del rol de la víctima en la sentencia, el de evitar el amparo de los delincuentes ante instituciones internacionales. Este soberanismo judicial se reivindica para delitos particularmente sensibles como son el terrorismo, la violación o el asesinato (Vox, 2019)Asimismo, en el ámbito de las inocuización o anulación del delincuente se opta por el rigor en el cumplimiento de las penas, en especial para delitos graves, contemplando la cadena perpetua. Por su parte, la dimensión de las penas agravadas tiene como ítem más relacionado la propuesta 92 al incidir en la eliminación de los privilegios penitenciarios, esta medida, cabe destacar, se aplicaría a penados por terrorismo y a inmigrantes ilegales.

Por lo que respecta al ámbito del electoralismo, esto se concentra en la medida contra la venta ambulante, que siendo un problema de escasa saliencia social, tenía presencia en la agenda mediática del momento. Además, sirve para reforzar la criminalización del inmigrante como elemento de riesgo y asociarlo, necesariamente, a actividades delictivas.

En relación con el temor a ser victimizado, la propuesta 86 alude a la regulación de ámbitos que generan alarma social. Esto está presente en el caso de la ocupación de viviendas y que inciden en situaciones de indefensión como la usura o en particular la posibilidad de resistencia o defensa. Estas son medidas que apelan fuertemente al sentimiento de seguridad de la población insider dentro del colectivo general de ciudadanos.

En cuanto a la dimensión de la criminalización, esta tiene dos elementos diferenciados. Uno, el que implica una asociación del inmigrante con situación irregular y por ello su relación con el rol de delincuente. El otro, es la asociación de profesar el credo musulmán con las actividades radicales y, en último término, terroristas. El ítem relacionado con la propuesta 31 tiene la finalidad de asociar, de manera directa, al extranjero con el delincuente de un modo casi categórico. 
Por último, la instrumentación del Código Penal se centra en tres ítems. El primero centrado en la propuesta 16, que pretende agravar la pena para tráfico de personas, e indirectamente está vinculada con la entrada de personas en situación irregular. Otra, es la que alude a la 30, con la tipificación del delito a la autoridad para proteger a funcionarios públicos en el ejercicio de sus funciones. Por último, la de introducir como delito el "despilfarro público", esta medida podría ir en las de tipo electoralista, dado que la corrupción es un problema sentido como relevante por la ciudadanía española. Además, no hay que olvidar que el partido matriz del que se escinde Vox estaba inmerso en procesos judiciales por corrupción en aquel momento.

\section{Tabla 6}

\section{Bloque populismo punitivo detallado Chega!}

\begin{tabular}{|c|c|}
\hline Bloque & Propuestas vinculadas \\
\hline Penalidad expresiva & - 19* castración química como pena acumulativa \\
\hline \multicolumn{2}{|l|}{ Protagonismo víctima } \\
\hline Inocuización & - 20 prisión efectiva \\
\hline Penas agravadas & $\begin{array}{l}\text { - } 23 \text { retirada de los beneficios penitenciarios } \\
\text { - } 24 \text { institución del trabajo en prisión. }\end{array}$ \\
\hline Electoralismo & $\begin{array}{l}\text { - } 25 \text { incautación de bienes delictivos para financiar cuerpos de } \\
\text { policía }\end{array}$ \\
\hline Temor a la victimización & - 31 regulación de la legítima defensa \\
\hline Criminalización & $\begin{array}{l}\text { - } 22 \text { publicación de nacionalidad y origen de los delincuentes } \\
\text { - } 58 \text { deportación de inmigrantes irregulares } \\
\text { - } 59 \text { inmigrante en situación ilegal dentro del país no podrá } \\
\text { regularizarse } \\
\text { - } 61 \text { pérdida de la nacionalidad para los inmigrantes por comisión } \\
\text { de delitos }\end{array}$ \\
\hline Prevención situacional & $\begin{array}{l}\text { - } 30 \text { dotación de medios para la vigilancia y control de las aguar } \\
\text { territoriales } \\
\text { - } 57 \text { replantear la política de fronteras en la UE }\end{array}$ \\
\hline Instrumentalización CP & $\begin{array}{l}\text { - } 19^{*} \text { castración química, registro de agresores } \\
\text { - } 21 \text { instauración de la cadena perpetua para delitos graves }\end{array}$ \\
\hline
\end{tabular}

Fuente: Elaboración propia.

La tabla 6 muestra los resultados para el caso de Chega! Un elemento destacable es que dentro de la literalidad de sus propuestas no existe un pro- 
tagonismo de la víctima como tal. Si bien la palabra figura tres veces a lo largo del manifiesto, no se encuadra dentro de las coordenadas con las que se entiende dentro del populismo punitivo. A pesar de todo, el programa del partido luso cubre (íntegramente) ocho de las nueve dimensiones constitutivas del populismo punitivo. Además, resulta reseñable los notables paralelismos respecto a su homóloga española.

En relación con la penalidad expresiva, uno de los ítems destacables está en la castración química para los penados por delitos de índole sexual, que además figura como pena acumulativa. Este tipo de delitos desata una fuerte corriente emocional y es particularmente sensible dentro de la opinión pública. En cuanto a la inocuización de los delincuentes, el ítem más asociado es el que aboga por el cumplimiento íntegro de penas para los delitos de violación. Esto se vincula con la siguiente dimensión, donde destacan dos ítems, por un lado, la retirada de los beneficios penitenciarios y, por otro, la instauración del trabajo penitenciario como mecanismo para que el penado financie su estadía en la cárcel.

La dimensión del electoralismo se centra, especialmente, en el ítem que implica la incautación de bienes ilícitos y el uso de su rendimiento para la financiación de agentes que han resultado heridos o con secuelas en cumplimento de su deber. Como se indica textualmente:

Modificar la legislación para permitir la clasificación de los bienes y valores que se acumulen para el Estado en los casos penales, para la constitución de un fondo permanente gestionado por parte de los representantes del cuerpo de policía con la finalidad de financiar a aquellos agentes que hayan resultado heridos físicamente en la lucha contra el crimen, o a los familiares directos de aquellos de dichos funcionarios. (Chega!, 2019, p. 4)

Esta medida se incardina como un mecanismo para solventar las situaciones de escasez de financiación y solvencia que atraviesan los cuerpos policiales portugueses.

Para la dimensión del temor a la victimización, el ítem es similar al mismo que figura en el caso de Vox, centrado en la juridificación y regulación de la legítima defensa de las personas que se ven en situaciones de victimización.

En la dimensión de la criminalización, la construcción del otro peligroso, es en general paralelo al que se ha presentado para la formación de la EDP española. Se concentra en la figura del inmigrante, en especial en situación de irregularidad, de ahí a la ilegalidad y, por ende, a la condición de de- 
lincuente. En paralelo, la dimensión de prevención situacional implica dos ítems, uno centrado en la dotación necesaria para la vigilancia de la frontera, en este caso marítima. Por otro, un replanteamiento de la política de fronteras e inmigración dentro de la Unión Europea, muy cercano, nuevamente, al planteamiento de Vox.

Por último, como elementos de instrumentalización del código penal, está la tipificación de la castración química, a la que se ha aludido con anterioridad. Además, destaca la introducción de la cadena perpetua para los delitos particularmente graves.

Como cierre a esta comparación, resta por señalar la existencia de varios paralelismos muy acusados entre ambas propuestas. Las propuestas 90 de Vox y la 21 de Chega! son prácticamente idénticas, al reivindicar la cadena perpetua para crímenes particularmente sensibles. Por otro lado, la propuesta 31 de la formación española es idéntica a la 22 de la lusa, aludiendo a la explicitación de la nacionalidad y origen de los delincuentes en las estadísticas criminales. Lo mismo ocurre en los casos de la medida 23 de Chega! y la 92 de Vox, que son prácticamente iguales, al solicitar la retirada de los beneficios penitenciarios para terroristas e inmigrantes ilegales, el enunciado literal es idéntico. Lo mismo ocurre en cuanto a las propuestas 58 (deportación de inmigrantes) y 59 (prohibición de regulación) de Chega! que son paralelas a las de Vox 14 y 15, para la primera, y a la 20 para la segunda. Todo esto no hace más que señalar las profundas concordancias entre ambas formaciones. Ello no resulta sorprendente dadas las similitudes socioeconómicas de ambos países vecinos y la situación parecida que han atravesado tras la Gran Recesión.

Cabe finalizar esta sección indicando que ambos partidos han puesto en práctica una estrategia de populismo punitivo a la luz de sus manifiestos electorales. En ambos casos, con un marcado sesgo antiinmigración. En el caso de la formación española resulta aún más notable y puede explicarse por la actividad en el pasado de la banda terrorista ETA como por los atentados islamistas que ha sufrido en su territorio. En uno y otro caso, se confirma la adopción por parte de ambas formaciones de una estrategia de populismo punitivo antiinmigración, o si se prefiere, de un populismo punitivo nativista. Este tipo de estrategia merece tal denominación, en tanto que parece estar latente la voluntad de ejercer de defensores de los ciudadanos nativos frente a la delincuencia y el crimen perpetrado preferentemente por extranjeros, para más señas, inmigrantes ilegales. 


\section{Discusión y conclusiones}

De acuerdo con los resultados expuestos en la anterior sección solo resta señalar que ambas formaciones de la EDP han empleado la estrategia del populismo punitivo dentro de los manifiestos electorales analizados. Además, con la particularidad que no solo lo han adoptado, además, lo han adaptado al discurso antiinmigración, propio de los partidos de esta naturaleza dentro de Europa.

El populismo punitivo nativista está presente en ambas formaciones, siendo un nexo de unión con el resto de fuerzas políticas de su ámbito ideológico en el Viejo Continente. De este modo, ambos partidos ibéricos se insertan en las tradiciones ya consolidadas de los partidos de extrema derecha europeos.

Cabe destacar, en todo caso, varias particularidades. En el caso español, el papel de la víctima como protagonista figura como una dimensión plenamente construida y explicitada. Una explicación es la larga actividad del terrorismo en el país, así como los atentados islamistas en su historia más reciente. Por el contrario, el caso portugués, donde este tipo de fenómenos no han ocurrido, el papel de la víctima está soslayado, queda implícito, permaneciendo más como una dimensión latente. El ciudadano insider se conceptúa como víctima potencial que debe protegerse, pero no se llega a reivindicar el protagonismo de la víctima como tal. Sin embargo, en uno y otro caso, a excepción del ya citado rol de la víctima, están presentes y completamente desarrolladas todas las dimensiones constituyentes del populismo punitivo como estrategia. Destacando siempre el modelo más completo de Vox, dado que tiene presentes y desarrollados todos los ítems o dimensiones que componen el populismo punitivo.

Por último, debe señalarse, para ambos casos, que el elemento crucial se encuentra en la construcción de un "otro peligroso" (dimensión de la criminalización), con un protagonismo absoluto del inmigrante como foco del riesgo. Por lo tanto, el sesgo nativista expresado en la idea de protección de los insiders nativos frente a los outsiders inmigrantes es un elemento constante en la construcción y adopción de la estrategia del populismo punitivo. Dado el crecimiento electoral de ambas fuerzas, no parece probable que en el futuro abandonen esta estrategia dentro de las propuestas para las políticas de seguridad y orden público, más bien al contrario. Esto es así dado que resulta una estrategia que, desde unas coordenadas nativistas, ha funcionado bien electoralmente en el resto de países europeos y parece que presenta el 
mismo resultado en la Península Ibérica. Por lo tanto, la estrategia del populismo punitivo nativista parece estar consolidada y adaptada en las formaciones de EDP dentro del espacio ibérico.

\section{Bibliografía}

Arrieta-Ruiz, Y. (2018). Punitive Populism and Symbolic Criminal Law. Inciso, 1(20), 37-45. https://bit.ly/2QeghW8

Berman, S. (2019). Populism is a Symptom Rather than a Cause. Polity, 51(4). http://dx.doi.org/10.1086/705378

Carrabine, E., Cox, P., Fussey, P., Hobbs, D., South, N., Thiel, D., \& Turton, J. (2014). Criminology. Routledge.

Chari, V., Dovis, A., \& Kehoe, P. (2020). Rethinking Optimal Currency Areas. Journal of Monetary Economics, 111, 80-94. https://bit.ly/3eejjls

Chega! (2019). 70 medidas para reeguer Portugal. https://bit.ly/3sx8IXR

Cohen, S. (2002). Folk Devils and Moral Panics. MacGiboo and Kee.

Cuervo-García, A. (2019). Perfil criminológico del delincuente de cuello blanco. En M. Bustos Rubio, \& F. Rodríguez Almirón, El sistema socioeconómico desde el prisma del derecho penal y la criminología (pp. 173-198). Aranzadi.

Davies, P., \& Jackson, P. (2008). The Far Right in Europe: An Encyclopedia. Greenwood World Publishing.

Díaz-Herrera, C. (2018). Investigación cualitativa y análisis de contenido temático. Orientación intelectual de revista Universum. Revista General de Información y Documentación, 28(1), 119-142. https://doi.org/10.5209/ RGID.60813

Díez-Ripolles, J. (2004). El nuevo modelo penal de la seguridad ciudadana. Revista electrónica de Ciencia Penal y Criminología (6). https://bit.ly/3n32duw

Esping-Andersen, G. (1995). The three worlds of welfare capitalism. Polity Press.

García-Pablos de Molina, A. (2014). Tratado de criminología. Tirant lo Blanch.

Garland, D. (2005). La cultura del control: crimen y orden social en la sociedad contemporánea. Gedisa.

Herrendorf, D.E. (1988). Revolución conservadora: consecuencias anti-sociales. RMCPS, 34(134),111-122. http://dx.doi.org/10.22201/fcpys.2448492xe. 1988.134.70830

Hough, M. (2008). Populism and Punitive Penal Policy. Criminal Justice Matters, 1(49), 3-5. https://doi.org/10.1080/09627250208553483 
Huguet -Santos, M. (2005). Reagan y el neoliberalismo europeo. En J.M. BeneytoPérez, A. Pérez-Sánchez \& R. Martín de la Guardia. Europa y Estados Unidos: una historia de la relación analítica en los últimos cien años (pp. 247-270). Biblioteca Nueva.

Larrauri, E. (2018). Introducción a la criminología y al sistema penal. Trotta.

Liang, C. (2007). Europe for the Europeans; The Foreign and Security Policy of the Populist Radical Right. En C. Liang, Europe for the Europeans; The Foreign and Security Policy of the Populist Radical Right (pp. 1-32). Aldershot.

Magone, J.M. (1999). Portugal: Paty System Installation and Consolidation. En M.D. David Brouhgton, Changing Party Systems in Western Europe (pp. 232-254). Cassel.

Martíns-Carvalho, P. (12-07-2017). Há minorias que se acham acima da lei. Temos tido excessiva tolerância. Noticias ao Minuto. https://bit.ly/2QgvNRt

Marx, I. (2020). The Working Poor. En B.G. (Ed.), Routledge International Handbook of Poverty (pp. 245-255). Routledge.

Mounk, Y. (2019). El pueblo contra la democracia. Paidós.

Mudde, C. (2019). The Far Right Today. Polity Press.

McLaughlin, E., \& Muncie, J. (2011). Diccionario de Criminología. Gedisa.

Nikolaus, P. (2010). La Gran Recesión: causas y efectos de la crisis que ha dislocado el mundo financiero y la economía cotidiana. Destino.

Picazo, A.J., \& Gil-Pareja, S. (2020). Sector servicios. En J.L. García-Delgado, \& R. Myro, Lecciones de economía española (pp. 235-254). Civitas.

Piketty, T. (2015). El capital en el siglo XXI. RBA.

Rísquez, M. (2016). Estrategia de devaluación interna y su impacto en la competitividad: España, 2008-2013. Investigación Económica, 75(297), 125-154. https://doi.org/10.1016/j.inveco.2016.08.004

Rodrik, D. (2012). La paradoja de la globalización: la democracia y el futuro de la economía mundial. Antoni Bosch.

Simón, A. (2005) La extrema derecha en Europa desde 1945 hasta nuestros días. Tecnos.

Stiglitz, J.E. (2003). El malestar en la globalizacion. Taurus.

Stiglitz, J.E. (2016). El euro: cómo la moneda común amenaza el futuro de Europa. Taurus.

Stojarová, V. (2018). Populist, Radical and Extremist Political Parties in Visegrad countries vis à vis the migration crisis. In the name of the people and the nation in Central Europe. Open Political Science (1), 32-45. https://doi. org/10.1515/openps-2018-0001 
Tejero-Pérez, A. (2017). Pobreza laboral en España. Un análisis dinámico. RIS, 76(2). https://doi.org/10.3989/ris.2018.76.2.16.54

Valles, M (1999). Técnicas cualitativas de investigación social. Síntesis.

Vox (2019). 100 medidas para la España viva. https://bit.ly/32ry8f1 